\title{
P04-2-58
}

Poster session

\section{VASORELAXANT EFFECT OF PROCYANIDIN B2 ON HUMAN INTERNAL MAMMARY ARTERY}

\author{
Aleksandra N Novakovic ${ }^{1}$, Marija Marinko ${ }^{1}$, Goran Jankovic ${ }^{1}$, Predrag Milojevic ${ }^{2,3}$, Ivan Stojanovic ${ }^{2,3}$, \\ Dragoslav Nenezic ${ }^{2,3}$, Vladimir Kanjuh ${ }^{4}$, Qin Yang ${ }^{5,6}$, Guo-Wei He \\ ${ }^{I}$ Department of Pharmacology, Faculty of Pharmacy, University of Belgrade, Serbia, ${ }^{2}$ Faculty of Medicine, University \\ of Belgrade, Serbia, ${ }^{3}$ Institute for Cardiovascular Diseases Dedinje, Belgrade, Serbia, ${ }^{4}$ Academy of Sciences and Arts, \\ Belgrade, Serbia, ${ }^{5}$ Department of Surgery, The Chinese University of Hong Kong, Hong Kong, ${ }^{6}$ TEDA International \\ Cardiovascular Hospital, Medical College, Nankai University, China
}

Introduction: Procyanidins are polyphenols abundantly found in plant food such as apples, grapes, nuts, cocoa, tea and red wine. Cardioprotective effect of procyanidins might be attributed, in part, to their vasorelaxant properties. The mechanism of vasorelaxation induced by procyanidin B2 is still unknown.

Aim: The aim of the present study was to investigate and characterize vasorelaxant effect of procyanidin B2 on human internal mammary artery (HIMA) as one of the mechanisms of its protective effect against vascular risk.

Material \& Methods: Discarded segments of HIMA were collected from patients undergoing coronary artery bypass grafting, transported to the laboratory and studied in organ baths. The HIMA rings were pre-contracted with phenylephrine.

Results: Procyanidin B2 induced strong concentration-dependent relaxation of HIMA rings pre-contracted by phenylephrine. Pretreatment with L-NAME, a NO synthase inhibitor, hydroxocobalamin, a NO scavenger, and ODQ, an inhibitor of soluble guanylate cyclase, significantly inhibited procyanidin B2-induced relaxation of HIMA, while indomethacin, a cyclooxygenase inhibitor, considerably reduced effects of low concentrations. Among $\mathrm{K}+$ channel blockers, iberiotoxin, a selective blocker of large conductance $\mathrm{Ca} 2+$-activated $\mathrm{K}+$ channels $(\mathrm{BKCa})$, abolished procyanidin B2-induced relaxation, glibenclamide, a selective ATP-sensitive K+ (KATP) channels blocker, induced partial inhibition, while 4-aminopyridine, a blocker of voltage-gated $\mathrm{K}+(\mathrm{KV})$ channels, and TRAM-34, an inhibitor of intermediateconductance $\mathrm{Ca} 2+$-activated $\mathrm{K}+(\mathrm{IKCa})$ channels, slightly reduced maximal relaxation of HIMA. Further, procyanidin B2 relaxed contraction induced by phenylephrine in $\mathrm{Ca} 2+$ free Krebs solution, but had no effect on contraction induced by caffeine. Finally, thapsigargin, a sarcoplasmic reticulum Ca2+-ATPase inhibitor, significantly reduced relaxation of HIMA produced by procyanidin B2.

Conclusion: These results demonstrate that procyanidin B2 produces endothelium-dependent relaxation of HIMA precontracted by phenylephrine. This effect is primarily the result of an increased NO synthesis and secretion by endothelial cells and partially of prostacyclin, although it involves activation of BKCa and KATP, as well as KV and IKCa channels in high concentrations of procyanidin B2. 